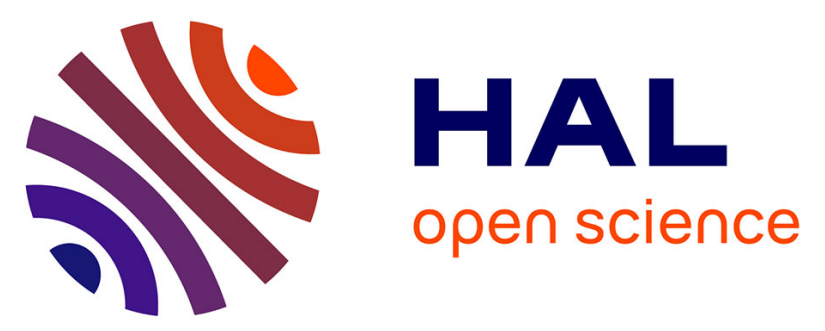

\title{
De la socialisation du consommateur à la participation aux activités de consommation: apports de la psychologie socio-historique et culturelle
} Valérie-Inès de La Ville, Valérie Tartas

\section{- To cite this version:}

Valérie-Inès de La Ville, Valérie Tartas. De la socialisation du consommateur à la participation aux activités de consommation: apports de la psychologie socio-historique et culturelle. Revue management \& avenir, 2011, Cahier: Vygotski aujourd'hui en management, 2011/2 (42), pp.131-149. 10.3917/mav.042.0133 . hal-01627854

\section{HAL Id: hal-01627854 \\ https://hal.science/hal-01627854}

Submitted on 14 Mar 2019

HAL is a multi-disciplinary open access archive for the deposit and dissemination of scientific research documents, whether they are published or not. The documents may come from teaching and research institutions in France or abroad, or from public or private research centers.
L'archive ouverte pluridisciplinaire HAL, est destinée au dépôt et à la diffusion de documents scientifiques de niveau recherche, publiés ou non, émanant des établissements d'enseignement et de recherche français ou étrangers, des laboratoires publics ou privés. 


\title{
De la socialisation du consommateur \\ à la participation aux activités de consommation : apports de la psychologie socio-historique et culturelle
}

\author{
From consumer socialization to \\ participation in joint consumption activities: \\ contributions from socio-historical and cultural psychology
}

\author{
Valérie-Inés de La Ville \\ Professeur de Sciences de Gestion \\ Directrice Centre Européen des Produits de l'Enfant \\ IAE - Université de Poitiers \\ Laboratoire Centre de Recherche en Gestion EA 1722 \\ Valérie Tartas \\ Maître de Conférences en Psychologie du développement \\ Université Toulouse 2 \\ Unité de Recherche Interdisciplinaire Octogone EA 4156 \\ Laboratoire Cognition Communication et Développement.
}

\section{Résumé :}

Cet article propose une analyse critique des perspectives utilisées dans la recherche en marketing pour modéliser le processus de socialisation des consommateurs. Pour pallier certaines limites inhérentes à ces approches, quelques fondements clés de la psychologie culturelle sont discutés afin d'éclairer la notion de participation à des activités conjointes de consommation et souligner que, dans le développement des compétences du consommateur, l'interaction avec autrui joue un rôle central, et que ce développement dépend de la transformation d'éléments institutionnels qui légitiment une culture de consommation historiquement située. (555 caractères)

Mots-clés : enfant consommateur - psychologie culturelle - socialisation économique recherche en marketing

\begin{abstract}
:
This article puts forward a critical analysis of the approaches used in marketing research to illustrate the consumer socialization process. To compensate for certain inherent limitations in these approaches, the basic foundations of cultural psychology are discussed to show how the notion of participation in joint consumption activities questions the classic view of socialization used in marketing, showing that interaction with others plays a central role in the development of consumer competences, and that this development is dependent upon the transformation of institutional structures which legitimize a historically situated consumption culture. (567 caractères)
\end{abstract}

Key words: child consumer - cultural psychology - consumer socialization - marketing research 


\section{Introduction : Comment devient-on un consommateur suffisamment compétent pour prendre part aux échanges marchands ?}

Le concept de socialisation du consommateur (consumer socialization) a été défini pour la première fois en 1974 par Scott Ward : "The processes by which young people acquire skills, knowledge and attitudes relevant to their functioning as consumers in the marketplace. » (Ward 1974 : 2) Cette définition volontairement large ouvre ce champ de recherches à des échanges interdisciplinaires afin de prendre en compte la complexité des contextes culturels et sociaux, les aptitudes langagières et motrices, le développement psychologique, les dimensions symboliques et émotionnelles qui entrent en ligne de compte pour être en mesure d'agir sur le marché. Comme le soulignent à la fois Ward et al. (1990) et Ekström (2010), la question de la socialisation du consommateur a privilégié les recherches sur les enfants en faisant l'hypothèse que la socialisation à la consommation au cours de l'enfance est déterminante et a un impact particulièrement fort sur les compétences de consommation développées par le jeune consommateur. En outre, cette définition met davantage l'accent sur les compétences que sur les processus (Kline, 2010) et reste attachée à une conception linéaire issue de la psychologie du développement en stades telle que proposée initialement par Piaget (Piaget, 1937/1967). Cependant les recherches montrent que ni l'âge ou le niveau scolaire, ni les influences externes telles que la famille, les médias, le groupe de pairs ne suffisent à expliquer comment l'enfant apprend à devenir un consommateur compétent (Ward et al., 1977).

Cette question - en apparence simple dans sa formulation - a ouvert tout un champ de recherches depuis le début des années 50 (Strauss 1952) qui ont abouti à des modélisations contrastées de la socialisation du consommateur. C'est pourquoi cet article se propose d'éclairer les fondements psychologiques de deux approches classiques de la socialisation du consommateur habituellement utilisées dans la recherche en marketing pour souligner à la fois leur contribution à la compréhension de ce processus complexe mais aussi leurs limites pour aborder certains types de questionnements ou d'enjeux liés au rôle du consommateur au sein du marché. Pour pallier certaines limites inhérentes à la notion de socialisation du consommateur, nous proposons de prendre appui sur quelques éléments clés de la psychologie socio-historique et culturelle pour formuler les bases d'une approche culturelle des activités ordinaires de consommation. En guise d'ouverture, nous esquissons les perspectives de recherche en marketing que ce cadre théorique est susceptible d'ouvrir.

\section{Le regard du marketing sur la socialisation du consommateur : limites et impasses...}

Dans cette première partie, nous ne ferons que rappeler très brièvement la synthèse de 25 années de recherche sur la socialisation du consommateur proposée par John (1999), dont nous avons déjà fait un commentaire critique (De La Ville \& Tartas, 2005). Nous traiterons à la suite une série de travaux en marketing qui cherchent à mettre en évidence l'impact des facteurs sociaux et techniques sur le processus de socialisation du consommateur.

Tout d'abord, John (1999), s'appuyant sur les stades du développement cognitif proposés par Piaget, détermine trois stades essentiellement définis en termes de tranches d'âge : le stade perceptuel (3 à 7 ans), le stade analytique (7 à 11 ans) et le stade réfléchi (11 à 16 ans). Cependant cette association directe des âges à des compétences cognitives et sociocognitives procède à notre avis d'un détournement des thèses piagétiennes. En effet, la notion de stade chez Piaget (voir 1937/1967, p. 217 pour un définition de cette notion ; Piaget et Inhelder, 1966) 
est bien plus complexe que ce qu'en retiennent les chercheurs en marketing en particulier parce qu'elle fait référence à des mécanismes cognitifs d'assimilation, d'accommodation et d'équilibration. Mais au-delà, cette modélisation en trois stades constitue bien plus un outil d'aide à la décision directement exploitable par les responsables marketing plutôt qu'une description fine et approfondie des connaissances réellement mobilisées par les enfants et les adolescents contemporains dans des activités de consommation. De ce fait, les critiques habituellement adressées au modèle piagétien s'appliquent également à ce modèle de socialisation du jeune consommateur : le sujet pris en compte est davantage un sujet épistémique - et non un sujet social - ce qui conduit à mettre en exergue l'incomplétude des compétences enfantines à propos des relations marchandes, en les décrivant comme uniquement perceptives dans un premier temps, égocentriques par la suite, et n'étant susceptibles de déboucher sur des modalités plus critiques qu'à l'adolescence. On retrouve ainsi le modèle de l'adulte raisonneur et logique, posé et socialisé aux règles du marché, considéré comme l'aboutissement du développement et donc comme étalon de comparaison avec un enfant en développement, et de ce fait forcément «incomplet » et non encore socialisé. Or les enfants contemporains, qui baignent dans une culture consommation de masse et un contexte de globalisation des média, acquièrent des connaissances et des modes de pensée extrêmement différents de ceux rapportés par ces recherches. Cram \& Ng (1999) notent que l'influence des processus d'interactions sociales, des contextes historiques, des attitudes politiques et sociales, du rôle des habitudes et des valeurs sur le développement de la socialisation économique de l'enfant ont été simplement négligés dans ces approches. L'ensemble de ces critiques s'appliquent au modèle de John dans lequel autrui joue un rôle limité à celui d'un facteur d'influence (ralentissant ou accélérant) sur le développement des compétences enfantines en matière de consommation, mais à aucun moment le rôle d'autrui, et plus largement de la culture, n'est constitutif du développement de l'enfant.

En synthèse, ce modèle recèle quatre limites majeures : l'enfant y est essentiellement décrit du point de vue de ses « incompétences » notamment avant l'adolescence, ses compétences en cognition sociale sont ainsi absentes de la littérature marketing sur la compréhension des intentions d'autrui, du mensonge, du raisonnement d'autrui, etc. (Deleau, 1999); seuls les facteurs internes sont évoqués pour rendre compte du développement en stades qui est conçu comme linéaire, ce qui conduit à retenir l'âge comme le facteur explicatif aux dépens de dimensions sociales et à négliger les différences qualitatives dans la nature du raisonnement à chaque stade ; la troisième limite tient au fait que seul le mode logique ou paradigmatique est observé pour expliquer l'apprentissage des conduites de consommation, or les travaux de Jérôme Bruner (1986/2000) notamment ont démontré que le mode narratif constitue une voie d'entrée dans la culture tout à fait essentielle bien que trop souvent négligée. La quatrième limite provient de la façon de définir le concept même de «socialisation » : il s'appuie sur la théorie piagétienne selon laquelle le développement de l'enfant consiste essentiellement en la socialisation progressive d'un individu à l'origine isolé, quasi " autiste » dans sa capacité à prendre en compte d'autres points de vue que le sien propre et le monde qui l'entoure. Ainsi parler de socialisation du consommateur avec comme soubassement épistémologique la théorie piagétienne (le plus souvent une approche piagétienne qui a été détournée car abusivement simplifiée) suppose de partir d'une conception de l'enfant comme un être initialement asocial qui au cours de son développement s'ouvrira progressivement au point de vue d'autrui. La socialisation apparaît donc comme une finalité du développement. Cependant, les recherches actuelles en psychologie du développement montrent que les enfants très tôt sont capables de prendre en compte autrui, de comprendre ses désirs, ses pensées, ses croyances, de s'adapter à leur partenaire selon les enjeux qu'ils perçoivent dans différentes situations de communication (Bruner, 1983 ; Deleau, 1990 et 1999 pour un aperçu des travaux sur la théorie de l'esprit). Il ne semble donc pas pertinent de réduire le développement uniquement à ce processus de socialisation. Ainsi si l'on considère les processus de socialisation et les processus 
d'individuation (c'est-à-dire de formation de la personne) comme deux versants indissociables et complémentaires du processus même du développement humain en suivant la proposition de Vygotski, nous serons amenées à redéfinir les processus de socialisation dans la troisième partie de cet article.

Pour rendre compte du développement de la socialisation économique de l'enfant, une autre série de travaux en marketing met l'accent sur les processus permettant à l'enfant d'acquérir les compétences économiques en soulignant qu'ils s'appuient sur un ensemble de facteurs sociaux (Moschis \& Churchill, 1978 ; Moschis \& Moore, 1979). Ainsi, le modèle proposé par Lassare et Roland-Lévy intègre quatre variables : les agents de socialisation (pairs, famille, école, mass media) ; les processus d'apprentissage (essentiellement l'imitation), les variables sociales structurales (âge, sexe, classes sociales) et le contenu d'apprentissage (Lassare \& Roland-Lévy, 1989; Roland-Lévy, 1990). Ce modèle constitue une synthèse entre trois approches distinctes : tout d'abord, la théorie piagétienne, reprise par Berti et Bombi (1989) pour le développement de la pensée économique qui retient le rôle actif de l'enfant dans la production de connaissances ; ensuite, le modèle des rôles sociaux, qui considère que l'enfant doit apprendre à jouer différents rôles dans la société, dont celui de consommateur apprentissage économique qui est en pratique effectué grâce à l'environnement familial et relationnel de l'enfant - ; et enfin, la théorie de l'apprentissage social de Bandura (1977) qui retient deux processus centraux - l'observation et l'imitation (des parents, pairs frères et sœurs, etc.) - pour expliquer à la fois les comportements de consommation concrets (comme comparer des prix et des produits) et les raisonnements sur des notions plus complexes (comme le pouvoir d'achat, les taxes).

Dans ces modèles, l'environnement social est représenté par deux variables clés : l'environnement social de l'enfant et la nature des feed-back et des renforcements produits par ce même environnement. Cependant, bien que le rôle d'autrui y soit vu comme plus central que dans les travaux précédents, d'un point de vue méthodologique, ce ne sont pas les interactions sociales qui sont au cœur de l'analyse. L'individu et l'environnement social sont conçus comme deux entités séparées qu'il convient de parvenir à réarticuler, mais l'individu demeure l'unité fondamentale d'analyse et de modélisation. Trois limites essentielles peuvent être relevées : premièrement, si l'apprentissage par observation et imitation constitue le processus retenu pour expliquer le développement de la socialisation économique des enfants, il n'apporte pas d'explication satisfaisante à l'apparition de comportements créatifs qui ne peuvent être assimilés à une simple imitation de comportements existant de façon préalable. En outre, ce modèle ne parvient pas non plus à expliquer ce qui se passe si les enfants sont exposés à des comportements moins compétents : les enfants régresseraient-ils ? Deuxièmement, ces études se centrent presque exclusivement sur les préadolescents ou adolescents en négligeant d'étudier les jeunes enfants, ce qui conduit à s'interroger sur les processus envisagés comme explicatifs du développement: ne sont-ils à l'œuvre qu'à un moment donné du développement? Troisièmement, le processus même de socialisation reste enraciné dans des soubassements théoriques envisageant un développement linéaire. Si ces modèles reconnaissent que l'environnement social a un impact sur les comportements de consommation, ils restent attachés à l'idée que le point d'achèvement du développement est l'adulte. Ces travaux de recherche en marketing intègrent une dimension sociale qui a l'avantage de conduire à une prise en compte de la culture dans laquelle évolue l'enfant, ce dernier n'étant plus considéré comme un sujet idéal, mais bien comme un enfant réel. Cependant cette dimension sociale ou la culture dans laquelle baigne le sujet demeurent des éléments contextuels qui se superposent au sujet, mais qui ne sont ni constitutifs ni productifs du sujet et de ses façons de se développer. 


\section{L'apport de la psychologie socio-historique et culturelle (Vygotski, Bruner) pour aborder le développement de l'enfant consommateur}

Au travers des limites de ces deux approches utilisées en marketing, la question de la prise en compte des actions des enfants en situation de consommation - c'est-à-dire des conduites « situées » et « distribuées » au sein de contextes communicatifs - apparaît comme centrale pour mieux comprendre comment l'enfant prend part à la culture de consommation de masse qui caractérise les sociétés occidentales. Nous proposons de reprendre trois éléments clés des théories qui attribuent au social - notion incluant à la fois les autres individus qui interagissent avec l'enfant mais aussi à l'usage qui est fait par l'enfant des instruments de la culture (Vygotski, 1934/1997) - un rôle constitutif dans le développement cognitif de l'enfant pour esquisser les bases d'une approche culturelle des pratiques de consommation auxquelles l'enfant en développement prend part. Nous rappelons dans un premier temps les trois niveaux clés proposés dans la psychologie de Vygotski et réagencés par Bronckart (2002) :

(a) la spécificité de l'environnement humain (c'est-à-dire définir les composantes qui le constituent),

(b) les processus de médiations socio-sémiotiques, et

(c) les processus d'appropriation et d'intériorisation constitutifs de la personne.

Ensuite, à partir de ces trois niveaux, nous développons un modèle des activités de consommation auxquelles participe l'enfant en développement.

La pensée de Vygotski, tout comme sa théorie, est complexe. L'objectif ici n'est pas de résumer sa pensée, tâche bien trop ardue ${ }^{1}$, mais de proposer, en reprenant quelques éléments clés de son approche, une nouvelle modélisation des enjeux liés à la socialisation du consommateur. Avant de présenter ces trois éléments clés, il est important de rappeler que pour Vygotski, le développement ne consiste pas essentiellement en «la socialisation progressive d'un individu à l'origine isolé et autiste, mais en l'individuation d'un organisme fondamentalement social dès l'origine » (Rivière, 1990, p. 73). Cette proposition de Vygotski invite clairement à concevoir autrement le développement de l'enfant consommateur jusque-là envisagé dans les deux cadres théoriques précédents comme une socialisation progressive et linéaire.

(a) Tout environnement est constitué, d'une part, du monde physique et d'autre part, surtout des comportements de nos semblables. Ce dernier aspect prend la forme d'activités collectives organisées orientées par différentes fonctions (au départ des fonctions de survie chez l'animal, qui chez l'homme, se sont détachées de cette finalité première et se sont complexifiées. Elles fournissent des cadres interprétatifs qui médiatisent désormais les rapports entre les hommes et le milieu physique). Ces activités utilisent ou s'appuient largement sur des outils et donnent lieu à la production d'objets sociaux et d'œuvres culturelles qui deviennent à leur tour des éléments de l'environnement général. Dans ce cadre théorique, la question centrale n'est plus de cerner une relation directe entre l'enfant et le produit, en termes d'étude des préférences du jeune consommateur par exemple, ni de décrire les facteurs susceptibles d'influencer son comportement de socialisation en tant que consommateur - la famille, les médias, les pairs, l'école, etc. - mais de prendre en compte les composantes essentielles de l'environnement particulier lié à une activité spécifique de consommation. Cette dernière constitue une activité pleinement sociale (car elle implique différents partenaires : l'enfant, autrui - incluant celui qui accompagne l'enfant tout comme

\footnotetext{
${ }^{1}$ Il existe de très bons ouvrages pour aller dans ce sens, par exemple : Rivière, 1990 ; Clot, 1999 ; Schneuwly et Bronckart, 1985
} 
celui qui a fabriqué le produit pour l'enfant -, médiatisée, c'est-à-dire qui pour se développer s'appuie sur divers instruments ou outils culturels (le langage oral et/ou écrit, l'ensemble des systèmes de signes utilisés dans cette situation) et qui se déroule dans un univers social, historique et culturel particulier (ensemble des valeurs, normes et conventions sociales et culturelles). Ainsi, dans les sociétés occidentales, on ne peut comprendre comment l'enfant entre dans la culture sans analyser les médiations sociales et sémiotiques qui rendent possible la participation de l'enfant à certaines activités de consommation.

(b) Ainsi, concernant les processus de médiations socio-sémiotiques en jeu dans les situations de consommation, il convient de préciser que l'enfant en développement est en cours d'apprentissage des règles et des enjeux de ce type d'activités. Ainsi, dans le cadre théorique proposé par la psychologie socio-historique, ces médiations sont définies comme fondatrices et constitutives du processus de développement de l'enfant. L'entourage de l'enfant joue un rôle décisif dans ce processus puisqu'il entreprend à l'égard de l'enfant des démarches délibérées de formation qui consistent à intégrer peu à peu l'enfant dans des activités conjointes (Bruner, 1991) ou de le faire participer à celles-ci (Rogoff, 2003). Les adultes commentent verbalement les activités auxquelles ils font participer l'enfant, permettant ainsi à ce dernier de focaliser son attention et ses actions sur des éléments clés de la situation. L'enfant est donc activement engagé dans une situation particulière grâce aux actions de l'adulte à son égard et aux explications qu'il reçoit concernant les règles sociales qu'il convient de prendre en compte.

(c) «Ainsi, dès la naissance, l'entourage propose à l'enfant des éléments d'informations issus de son histoire culturelle ou met à sa disposition des éléments de représentations collectives dans le cadre de productions langagières associées à des activités 'pratiques'. » (Bronckart, 2008, p. 239). Les façons de penser, de faire, de donner du sens propres à l'enfant - qui est ainsi amené à faire fonctionner ces outils en pratique - peuvent s'en trouver potentiellement transformées (Rogoff, 1998). En principe, comme l'affirme notamment Vygotski (1934/1997), cette transformation résulte de l'intériorisation des signes langagiers (dans leurs rapports aux activités collectives et aux connaissances formelles), tels que l'entourage les présente dans ses démarches de formation et d'accompagnement de l'enfant (Rogoff et Wertsch, 1984). Ainsi, utiliser cette perspective pour comprendre le développement de l'enfant dans la société de consommation contemporaine, conduit à éclairer explicitement non seulement la façon dont l'enfant est conduit à s'engager lui-même dans ces activités de consommation, mais aussi les conditions et la nature même des réorganisations cognitives et comportementales engendrées par les différentes modalités de participation à ces activités. Ce qui permettra alors de comprendre en quoi certaines réorganisations peuvent s'avérer être négatives, ou non développementales en quelque sorte, et d'autres, au contraire, peuvent générer des réorganisations positives ou du développement, et accroître les compétences de cet apprenti-consommateur.

Dans la perspective de Vygotski, l'accent est donc mis sur les médiations sociales et sémiotiques pour comprendre et expliquer non seulement le développement des fonctions psychiques supérieures (mémoire, attention, activités de conceptualisation, etc.), mais également comment les différentes formes d'activités collectives ou sociales sont à la fois constitutives et productives de ces formes de pensée. C'est là l'originalité de l'approche développementale de Vygotski. De quoi les activités de consommation auxquelles l'enfant est engagé à participer sont-elles constitutives et productives? Ou formulé autrement, à quels types d'activités de pensée donnent-elles forme ? En outre, une autre question centrale que soulève 
la perspective de Vygotski est celle du rôle de l'éducation ou des situations d'enseignementapprentissage : l'éducation de l'enfant à la consommation doit-elle être envisagée comme une co-éducation portée à la fois par les parents, les éducateurs et les professionnels du marketing qui fournissent des outils culturels à l'enfant pour prendre part aux activités de consommation? Cette perspective théorique et ses prolongements contemporains notamment chez Bruner et Rogoff peuvent-ils nous aider à proposer un nouveau modèle pour penser les activités de consommation auxquelles participent les enfants? Voilà le défi que nous proposons d'esquisser, à défaut de réellement parvenir à le relever, dans la dernière partie de cet article.

\section{Devenir un consommateur à travers différentes modalités de participation à des activités conjointes de consommation}

Les éléments qui précèdent nous permettent d'aborder sous un jour nouveau la question du développement de l'enfant en tant qu'apprenti-consommateur. Nous posons dans cette partie les fondements de la modélisation d'inspiration socio-historique que nous proposons pour comprendre la façon dont l'enfant développe des compétences de consommateur et nous en illustrons certains points à travers diverses situations de consommation auxquelles l'enfant prend part. (cf. Figure 1). Notons qu'une interaction inverse et complémentaire de celle que présente la Figure 1 peut être également envisagée, illustrant comment l'objet constitue un médiateur de la relation à autrui. Dans cette perspective, les travaux de Nadel et Baudonnière (1985) analysent le rôle de l'objet médiateur de la communication avec autrui chez les jeunes enfants et ceux de Blandin (2002) détaillent comment les objets contribuent à structurer les relations sociales entre un sujet et un autrui généralisé en fonction d'une pluralité de registres - utilitaire, symbolique, mémoriel, sémiotique, marchand, etc.

Tout d'abord, l'enfant n'est jamais seul face à un produit ou un service marchand : il est toujours guidé, de façon directe ou indirecte, par autrui, qu'il s'agisse de sa mère pour le nourrisson, de ses parents ou de sa fratrie pour le jeune enfant, ou de ses copains pour l'enfant scolarisé, de ses «amis» pour l'adolescent féru de réseaux socio-numériques, dans sa découverte active des fonctionnalités du produit, de ses usages potentiels et des point de repère clés nécessaires pour en maîtriser la dépense induite.

Ensuite, l'intérêt que porte un enfant à un produit ou service marchand n'est jamais purement spontané ou inné : l'intérêt que l'enfant éprouve pour une offre commerciale ne provient pas d'une structure interne de préférences pré-existante et plus ou moins stabilisée, mais est principalement dû à l'histoire sociale et institutionnelle de ce produit ou service, aux occasions de sa consommation, à l'imaginaire culturel et aux croyances qui lui sont associés dans une société donnée. A travers un répertoire linguistique et un ensemble de signes particulièrement développé à propos de certaines activités de consommation, c'est l'entourage de l'enfant - parents, grands-parents, éducateurs, fratrie, copains - qui canalise en grande partie les centres d'intérêt de l'enfant. Par exemple, dans une famille férue de nouvelles technologies car le père ou la mère sont ingénieurs, l'enfant va être naturellement conduit à utiliser de façon précoce la télécommande pour jouer à des jeux vidéo ou utiliser l'ordinateur ou bien aller surfer sur Internet. A la fois les concepts liés aux nouvelles technologies mais aussi les gestes et les points de repère clés sur les bons usages et les bons réflexes - de prudence ou de modération vont lui être inculqués peu à peu à travers une initiation au quotidien qui va lui permettre d'accroître progressivement sa compétence de consommateur. Ainsi, chaque famille a une histoire propre qui la conduit à privilégier des aspects esthétiques ou bien sensoriels ou encore intellectuels dans les choix de consommation qu'elle opère dans différentes activités de consommation. Il faut bien comprendre qu'au-delà de la famille ou des cercles de relations dans lesquels évolue l'enfant, les techniques marketing participent également de cette transmission 
culturelle à propos des produits et services qui, permet de focaliser l'intérêt de l'enfant sur certaines catégories de produits et services aux dépens d'autres. Ainsi, de façon variable selon les sociétés, l'accès ou l'interdit lié à certains types de produits ou de services constitue un marqueur de rites sociaux comme l'anniversaire, ou religieux comme la première communion, ou de rites de passage comme la puberté, l'accès à la majorité, etc. Ce qui démontre que la signification réelle du produit ou du service, et donc sa valeur pour l'enfant, ne se révèle qu'à travers les règles culturelles, sociales et institutionnelles de son usage. C'est pourquoi, même si elle se donne à voir parfois de façon solitaire par un accès autonome de l'enfant à certains produits rapportés par autrui du supermarché ou par des dépenses personnelles d'argent de poche, la consommation de l'enfant est toujours une activité conjointe qui fait référence à un collectif, à un groupe qui a contribué en amont à focaliser l'intérêt de l'enfant sur le produit ou le service en lui procurant le vocabulaire et le système de valeurs indispensables pour faire sens de l'activité de consommation.

Figure 1. L'activité conjointe au cœur des pratiques de consommation enfantine

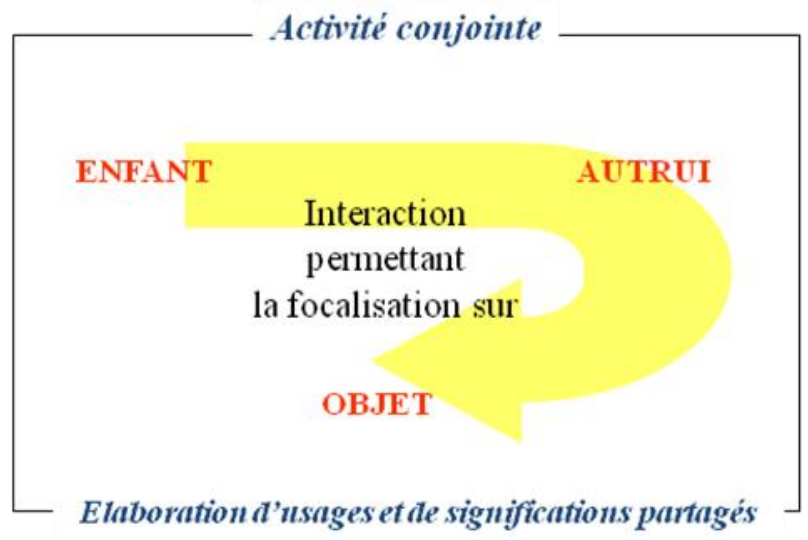

Dans ce cadre, la socialisation du jeune consommateur se pose en des termes plus larges : pour donner du sens à ses pratiques de consommation et les faire évoluer, quel usage l'enfant fait-il des outils culturels qu'on lui transmet ? Rogoff et ses collaborateurs affirment que «mettre l'accent sur l'organisation de la participation enfantine dans les pratiques culturelles, conduit à porter l'attention à la fois sur le rôle de guide des traditions culturelles et sur le rôle actif des individus eux-mêmes. » (Rogoff et al., 2007: 104) ${ }^{2}$ Ce qui suppose de centrer l'analyse sur l'effet combiné des médiateurs culturels qui structurent l'expérience de consommation telle qu'elle est vécue par l'enfant : le langage, le cercle familial, le groupe de pairs, les systèmes techniques et médiatiques auxquels l'enfant a accès, les techniques marketing qui sont mobilisées par les entreprises (design, packaging, communication publicitaire, aménagement de l'espace commercial, etc.), l'institution éducative qui a la charge d'initier l'enfant aux bonnes pratiques de consommation à travers des initiatives comme l'éducation nutritionnelle promue par le $\mathrm{PNNS}^{3}$ dans les écoles primaires ou la préparation au $\mathrm{B} 2 \mathrm{i}^{4}$ pour le collégiens.

En ouvrant tout un pan nouveau de questionnement et de recherche, la psychologie sociohistorique invite à une observation fine des pratiques à travers lesquelles l'enfant apprend à se comporter comme un consommateur. Quels sont les médiateurs culturels à l'œuvre lorsqu'un enfant reconnaît un produit ou un service comme lui étant assigné (forme, présence de mascottes, logotype, texture, sonorités, goût, caractère ludique de la narration publicitaire...)? De quelle façon un enfant apprend-il à faire un usage socialement acceptable d'un produit ou

\footnotetext{
${ }^{2}$ Article original : Rogoff, B., Moore, L., Najafi B., Dexter, A., Correa-Chavez M., Solis, J. (2006). Children's development of cultural repertoires through participation in everyday routines and practices, in Grusec, J. \& Hasting, P. (Eds.). Handbook of Socialization, N.Y.: Guilford.

${ }^{3}$ Programme National Nutrition Santé : http://www.mangerbouger.fr

${ }^{4}$ Brevet Informatique et Internet : http://www.educnet.education.fr/formation/certifications/b2i
} 
service en distinguant les différents contextes de son usage (avec ses parents, sa fratrie, ses copains, ses amis sur les réseaux socio-numériques, etc.) ? Quels instruments culturels les apprentis-consommateurs mobilisent-ils en vue d'effectuer des arbitrages quand ils sont confrontés à des difficultés pratiques liées à l'achat ou à la consommation de certains produits ou services ? Comment évoluent les capacités de négociation du jeune consommateur dans le but d'obtenir le produit ou service qu'il convoite mais pour l'achat duquel son autonomie n'est pas jugée encore suffisante par son entourage ? Comment l'enfant va-t-il prendre en compte différents contextes pour adapter la formulation et la justification de sa demande (récompense en fonction de résultats scolaires, expression du lien affectif entre parent et enfants ou entre pairs, produit présenté comme spécialement adapté aux enjeux de développement des enfants, produit emblématique de ce qui définit l'enfance ou la jeunesse dans la société contemporaine, etc.).

Cette perspective culturelle suppose également de comprendre comment l'enfant devient capable de discriminer les contextes de signification dans lesquels se déroule l'activité conjointe de consommation, et ainsi de distinguer les communautés de pratiques auxquelles il doit se référer pour y ajuster son niveau d'engagement : "l'enfant assimile les différentes formes de participation en fonction des différentes traditions dans lesquelles il est engagé de façon routinière. Quand les enfants traversent des lieux impliquant différents formats de participation, ils s'y engagent activement en fonction de leur propre répertoire de pratiques. Ce concept permet de mettre l'accent sur la puissance d'agir (agency) propre à l'enfant dans sa manière de sélectionner, rejeter ou transformer les multiples façons de s'engager dans le monde. » (Rogoff et al., 2007: 105) ${ }^{5}$ Ce passage souligne la capacité créative de l'enfant qui joue avec les règles qu'on lui impose, mais parvient parfois à les détourner, à les aménager pour rendre son quotidien, très fortement contraint par les décisions des adultes, tout simplement vivable de son point de vue.

En synthèse, nous pouvons dire qu'un modèle non pas de socialisation économique de l'enfant mais de participation aux activités de consommation s'appuyant sur les fondements de la psychologie historico-culturelle et culturelle permet de pallier certaines limites des deux approches jusqu'à présent mobilisées dans les recherches en marketing. La notion de participation de l'enfant à des activités conjointes de consommation souligne le rôle clé d'autrui et de la culture de consommation de masse contemporaine pour le développement des connaissances mobilisées par cet apprenti-consommateur. En outre, elle invite à tenir compte de l'évolution progressive des éléments institutionnels qui rendent possible et légitiment la place de l'enfant au cœur même des relations marchandes. Dans ce cadre, l'enjeu clé pour le responsable marketing consiste à susciter la transformation de la participation de l'enfant à différentes activités conjointes de consommation. Pour reprendre les apports de Lave et Wenger (1991), c'est en participant à des activités sociales situées culturellement - telles que l'apprentissage du métier de tailleur, de celui de maître de quart dans la marine américaine ou les réunions d'entraide des alcooliques anonymes abstinents... auxquelles nous proposons de rajouter les activités de consommation, que l'apprenant observe puis s'approprie progressivement des pratiques en s'immergeant dans une communauté de pratiques. Les techniques marketing, tout comme les autres médiateurs langagiers, symboliques et physiques, accompagnent l'apprenti-consommateur pour le conduire progressivement à passer d'une participation périphérique - comme utilisateur d'un produit acheté par ses parents, observateur de situations d'achat, etc. - à une participation centrale - en tant que décideur autonome sur certaines catégories d'articles, expert reconnu de certains types de produits... (La Ville et Tartas, 2008) La problématique révélée par cette approche met en lumière les défis auxquels est confronté le marketing en pratique : d'une part, les techniques marketing peuvent contribuer à surmonter la résistance des enfants afin d'étayer le projet éducatif des parents ; d'autre part,

\footnotetext{
${ }^{5}$ Ibidem.
} 
ces mêmes techniques, en instituant l'enfant comme décideur de ses propres pratiques de consommation, sont porteuses de risques tels que des formes de transgressions ou l'invention de nouvelles pratiques partiellement détachées des normes sociales en vigueur...

\section{Transformer la participation de l'enfant aux activités conjointes de consommation : perspectives de recherche...}

Approfondir les fondements psychologiques mobilisés dans les recherches en marketing sur la socialisation du consommateur conduit à la nécessité de distinguer les soubassements épistémologiques de quatre projets de connaissance.

D’une part, en psychologie du développement, le processus même de socialisation est conçu dans des perspectives contradictoires :

- Certaines modélisations (John 1999 ; Lassare et Roland-Lévy 1989) reposent sur l'idée d'un accroissement de la socialisation au cours du développement : l'enfant passe d'une étape égocentrique à une étape décentrée ou il devient alors capable de prendre en compte le point de vue de l'autre et d'anticiper les conséquences de sa propre conduite sur le point de vue de l'autre. La socialisation - notion qui dans ce cas ne recouvre que la socialisation relationnelle - est conçue comme un point d'achèvement du développement de l'enfant qui raisonne enfin de façon comparable à l'adulte.

- D'autres travaux en psychologie, et en particulier l'ensemble des recherches développées dans le cadre épistémologique mis en forme par Vygotski et ses collègues, considèrent que la socialisation peut être envisagée comme centrale si elle recouvre non pas uniquement la socialisation relationnelle mais si elle intègre également le social dans une acception plus large : la dimension sociale n'est pas uniquement constitutive des modes de pensée, car elle doit être comprise comme un élément clé de production de ces modes de pensée. Elle doit par conséquent être envisagée comme un processus permanent et crucial du développement revêtant différentes formes (relationnelle, sémiotique, institutionnelle, historique, etc.).

D'autre part, dans le champ des recherches en marketing, l'activité de consommation est conçue à travers deux perspectives contradictoires :

- Une première série de travaux largement rencontrée dans les recherches sur les préférences du consommateur et les choix impliqués dans les comportements d'achat, n'a retenu dans l'activité de consommation que le processus rationnel de prise de décision d'achat.

- D'autre part, voulant dépasser cette première approche trop directement inspirée des théories rationnelles de la décision, les tenants du marketing expérientiel ont proposé d'intégrer des dimensions affectives et symboliques aux processus de prises de décision impliqués dans les activités de consommation (Hirschman et Holbrook 1992 ; 1995 ; Oestergaard et Jantzen, 2000). Ensuite, les tenants du courant de la Consumer Culture Theory (Arnould et Thompson, 2005) vont encore plus loin en défendant l'idée que la consommation constitue une expérience culturelle signifiante grâce à laquelle l'individu construit en partie son identité. Si elle ne revendique pas de filiation directe avec le cadre épistémologique et théorique élaboré par Vygotski et ses épigones tels Rogoff ou Lave et Wenger, il semble que cette deuxième perspective puisse offrir des points d'articulation avec la psychologie socio-historique et culturelle. 
En croissant ces deux axes, nous pouvons proposer une représentation du champ scientifique de la socialisation du consommateur selon quatre projets scientifiques contrastés (cf. Figure 2)

L'enfant comme un apprenti acheteur rationnel : cette perspective étudie de façon exclusive le développement de la pensée logico-mathématique chez l'enfant et souligne que, par comparaison avec la façon dont les adultes raisonnent, les processus cognitifs chez l'enfant sont incomplets et fragiles, manquent de cohérence et de stabilité. L'étude de la socialisation de l'enfant consommateur a été structurée de façon spéculaire par rapport à l'adulte, ce qui a conduit à souligner les limites cognitives, langagières et comportementales de l'enfant par comparaison avec l'adulte. Par exemple, le champ sémantique restreint que maîtrise l'enfant ne lui permet pas de formuler de façon suffisamment précise ses préférences, ces dernières étant plutôt fondées sur un processus de mémorisation orale ou visuelle ou encore sur des réactions émotionnelles.

L'enfant comme un utilisateur découvrant les dimensions symboliques des produits: cette approche va plus loin en ce qu'elle inclut la dimension symbolique associée aux produits ou services marchands. Ce qui conduit à souligner que l'enfant doit parvenir à maitriser les caractéristiques culturelles des produits de façon à devenir un consommateur autonome aussi compétent que l'adulte. Toutefois le processus d'apprentissage y est dépeint comme individuel et prenant corps à travers des comportements d'imitation et l'exposition à des modèles de rôles sociaux, ce qui conduit à ignorer le fait que la consommation n'est pas seulement une question de répétition pertinente de comportements observés dans différentes situations sociales, mais consiste parfois en une invention collective.

Figure 2. Quatre projets scientifiques sur la formation des compétences de consommation 


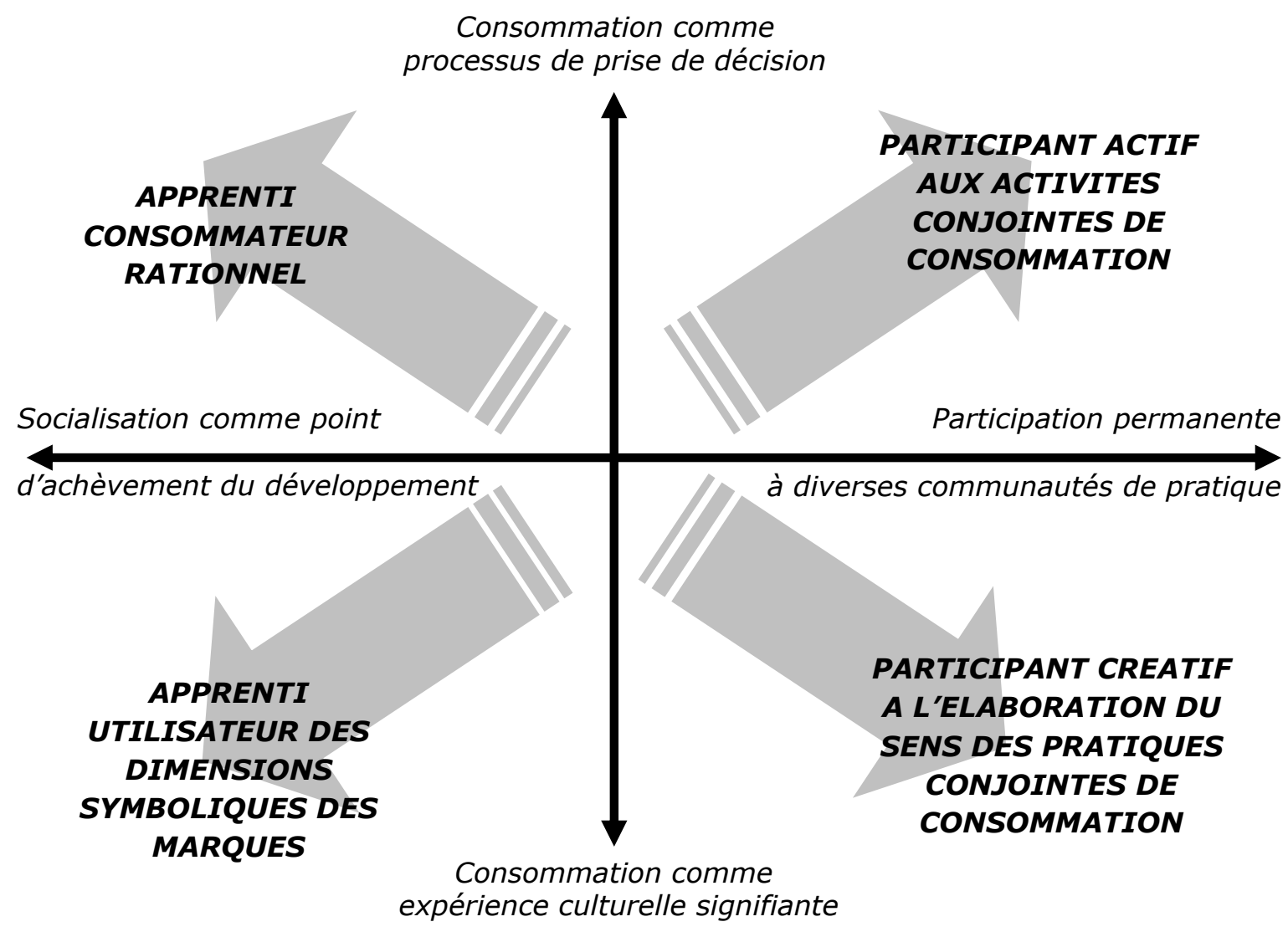

L'enfant comme un participant actif au sein d'activités conjointes de consommation: cette perspective souligne que l'enfant n'entre jamais seul ni directement dans le champ des pratiques marchandes. La participation à la société de consommation s'opère à travers une longue période d'apprentissage et de formation pendant laquelle l'enfant passe d'une situation de dépendance totale - en particulier à l'égard de ses parents - à la construction progressive de formes d'autonomie lui permettant de développer ses propres pratiques de consommateur. Ainsi, la consommation enfantine doit être conçue comme une activité pleinement sociale puisque l'enfant ne se trouve jamais livré à lui-même, mais il est guidé et supervisé par autrui pour apprendre à aborder et traiter un certain nombre de situations pratiques. De ce fait, l'unité d'analyse pertinente n'est certainement pas l'enfant conçu comme une monade isolée, mais plutôt l'activité conjointe menée dans le cadre d'une interaction 'adulte - enfant' ou "enfant plus expérimenté - enfant" ou un petit groupe confronté à un problème pratique de consommation (décision d'achat, choix entre différents produits, usage du produit, etc.) en s'inspirant des propositions vygotskiennes.

L'enfant comme un participant créatif dans l'élaboration du sens : cette approche conçoit l'enfant comme un agent social pleinement compétent - c'est-à-dire ayant ses propres compétences et capable de faire sens des différentes situations conjointes de consommation auxquelles il participe avec ses parents, sa fratrie, ses éducateurs, ses pairs, etc. La capacité créative des enfants est mise en exergue puisqu'on leur reconnaît la capacité de concevoir leurs propres activités et aussi de structurer activement leurs propres significations culturelles qui parfois démontrent des formes de résistance aux pressions normatives des adultes. Le programme d'activités des enfants et les enjeux qui y sont associés diffèrent de celui des adultes, parents, éducateurs et même professionnels du marketing qui s'adressent à eux. En conséquence, le fait de reconnaître que les enfants ont une capacité d'agir qui leur est propre dans différentes activités de consommation conduit à questionner les sources de vulnérabilité de l'enfant consommateur. Le risque se situe peut-être moins en termes d'immaturité ou 
d'incomplétude des processus cognitifs de l'enfant, et peut-être davantage dans les cadres culturels 'non conventionnels' ou 'détournés' que l'enfant est susceptible d'appliquer pour faire sens d'une activité conjointe de consommation située.

Ces quatre orientations structurent des programmes de recherche très contrastés pour aborder la question de la socialisation du consommateur, qui permettent de redonner à l'enfant une place au sein des théories de la consommation (Cook 2008). Considérer que la socialisation du consommateur constitue l'aboutissement d'un processus linéaire de développement de compétences, conduit à considérer que l'adulte est un consommateur compétent à titre individuel quelle que soit l'activité de consommation considérée, ce qui est bien évidemment très discutable. Considérer en revanche que la socialisation constitue un processus permanent à travers lequel l'individu est conduit à faire évoluer sa participation à des activités conjointes de consommation permet de souligner que les compétences individuelles se construisent d'abord au sein d'interactions et que ces dernières peuvent conduire à des comportements, des raisonnements et des systèmes de valeurs innovants. Ainsi, la psychologie socio-historique et culturelle fournit des soubassements épistémologiques et théoriques indispensables pour comprendre comment se forment les compétences du consommateur dans une société donnée en invitant à prendre en compte les éléments institutionnels qui contribuent à définir et à justifier la finalité, les situations et les conditions de la participation de l'enfant comme de l'adulte à une pluralité d'échanges marchands (La Ville et Tartas 2010).

\section{Conclusion : Apprendre à consommer, défis et perspectives...}

Cet article a discuté de façon approfondie les fondements psychologiques des deux principales perspectives utilisées dans la recherche en marketing pour modéliser le processus de socialisation des consommateurs : un modèle en stades du développement cognitif ainsi qu'une perspective processuelle qui visent à décrire comment l'enfant acquiert des connaissances économiques. Les limites inhérentes à ces approches justifient l'intérêt d'étudier la façon dont les enfants participent à la culture de consommation de masse en s'appuyant sur les acquis de la psychologie socio-historique et culturelle. Dépasser ces deux premières perspectives paraît incontournable pour développer une perspective située basée sur le comportement in situ des acteurs en présence (enfant-enfant- objet convoité/ enfant-parent-objet à acheter/objet acheté et utilisé etc.) dans la société contemporaine afin de comprendre et analyser les processus en jeu dans leur diversité et non uniquement à travers le prisme d'une socialisation réduite à ses aspects relationnels mais détachée de ses fondements institutionnels et idéologiques.

L'apport de l'œuvre de Lev Vygotski ouvre en particulier trois défis majeurs :

- L'importance des médiations verbales, sémiotiques dans les activités de consommation et des médiations sociales : il parait intéressant et novateur pour comprendre le développement de l'enfant de façon non linéaire et non normée, de bien saisir les contextes sociaux et culturels saturés d'outils culturels (jeux vidéo, internet, jouets, livres, messages marketing, images publicitaires, espaces commerciaux virtuels et réels, etc.) dans lesquels évoluent les enfants contemporains ainsi que de se donner les moyens d'étudier les conditions de leur participation aux activités complexes de consommation et d'usage de ces outils. Ce qui suppose de faire preuve d'inventivité sur le plan méthodologique en privilégiant des approches indirectes - à travers un discours réflexif, par l'auto-confrontation avec sa pratique, etc. - de la compétence de l'enfant en situation de consommation.

- Questionner notre rôle en tant qu'adulte auprès des enfants en cours de développement dans une société de consommation de masse - ou dans une société en développement : le rôle de 
l'éducation est fondamental dans la perspective vygotskienne qui distingue deux « formes » de transmission éducative : les contextes informels d'enseignement / apprentissage (par exemple, les apprentissages qui se réalisent dans les contextes familiaux, entre pairs dans des activités qui ne sont pas tournées systématiquement vers la transmission de savoir-faire et de connaissances etc.). Ces apprentissages informels se réalisent principalement au cours des expériences quotidiennes, au cœur de pratiques sociales ordinaires. L'enfant dans ces situations sociales n'a pas conscience des opérations qu'il effectue. C'est au cœur des contextes formels d'apprentissage dans lesquels l'objectif même des activités qui s'y déploient est d'apprendre à l'enfant à raisonner au-delà de ce qu'il sait faire ici et maintenant que l'enfant est alors amené à résoudre des problèmes nouveaux, qu'il ne rencontre pas dans son expérience quotidienne en-dehors de l'institution scolaire. Il s'agit, avec l'aide de l'enseignant, d'apprendre des connaissances élaborées dans un domaine de connaissance spécifique, ce qui permet à l'enfant de prendre conscience de ses propres opérations de pensée et de développer sa réflexivité sur ses propres pratiques. Qu'en est-il en matière d'éducation à la consommation ? Comment les enfants apprennent-ils de façon informelle des connaissances économiques, à travers leur participation à l'activité ordinaire des courses ? Comment l'institution scolaire / se saisit-elle du devenir de l'enfant dans une société de consommation de masse?

- Le modèle de développement proposé par Vygotski ne postule pas de différence ontologique entre l'enfant - qui apprend - et l'adulte - qui sait déjà (Wertsch, 1985). En effet, la figure 2 peut être indifféremment lue pour l'enfant ou pour l'adulte, ce qui incite à nuancer le propos et à étudier dans quels contextes l'adulte se trouve aussi en situation de développement de ses compétences de consommateur. Les soubassements conceptuels de la psychologie sociohistorique et culturelle permettent d'intégrer dans un même cadre théorique le processus de socialisation inversée (Gollety 1999 ; Ekström 2007) où l'adulte est guidé par un enfant ou un autre adulte - ayant une expertise supérieure sur l'activité de consommation considérée, comme par exemple établir son profil sur un réseau socio-numérique...

\section{Bibliographie}

Arnould E.J., Thompson C.J. (2005), « Consumer Culture Theory (CCT): Twenty Years of research », Journal of Consumer Research, 31, 868-882.

Bandura A. (1977), Social learning theory. Englewood Cliffs, N.J., Prentice Hall.

Berti A., Bombi A.S (1988), The child's construction of economics. Cambridge, MA: Cambridge University Press.

Blandin, B. (2002), La construction du social par les objets. Paris : Presses Universitaires de France, Sociologie d'aujourd'hui.

Bronckart J-P. (2002), «Les processus de socialisation. Le déterminisme culturel et son dépassement » - In : III Conference for Sociocultural Research : New conditions for knowledge production : globalization and social practices. CD-Rom, Universidade Estadual de Campinas, $28 \mathrm{pp}$.

Bronckart J-P. (2008), «Un retour nécessaire sur la question du développement »- In : M. Brossard \& J. Fijalkow (Eds.), Vygotski et les recherches en éducation et en didactiques, (pp. 237-250). Bordeaux : Presses Universitaires de Bordeaux. 
Bruner, J. (1983). Savoir faire, savoir dire. Paris : Presses Universitaires de France.

Bruner J. (1986/2000), Actual Minds, possible worlds. (trad. Française: Culture et mode de pensée: l'esprit humain dans ses æeuvres). London : Harvard University Press.

Bruner J. (1991), Acts of Meaning. London: Harvard University Press.

Clot Y. (1999), Avec Vygotski. Paris: La dispute.

Cook D.T. (2008). The missing child in consumption theory, Journal of Consumer Culture, 8 (2), 219-243.

Cram F., Ng S.H. (1999), «Consumer Socialization », Applied Psychology: An international review, 48(3), 297-312.

De La Ville V.I., Tartas V. (2010), Developing as Consumers, Chapter 2 in Marshall D. (Ed.), Understanding Children as Consumers (pp. 41-60). London: Sage.

De La Ville V.I., Tartas V. (2008), Transformer la participation de l'enfant aux activités de consommation alimentaire Enfance, 3, Numéro thématique «L'enfant face aux aliments », 299307.

De La Ville V.I., Tartas V. (2005), L'activité de consommation enfantine et ses médiateurs, in De La Ville V.I. (Ed.), L'enfant consommateur (pp. 73-88). Paris : Vuibert.

Deleau, M. (1990). Les origines sociales du fonctionnement mental. Paris : Armand Colin.

Deleau M. (Coord.) (1999), Psychologie du développement. Paris: Bréal.

Ekström K.M. (2007), Parental consumer learning or "keeping up with the children", Journal of Consumer Behavior, 6, 203-217.

Ekström K.M. (2010), Consumer socialization in families. In Marshall D. (Ed.), Understanding Children as Consumers, (pp. 41-60). Sage, London.

Gollety M. (1999), Lorsque parents et enfants s'apprennent mutuellement à consommer..., Décisions Marketing, 18, 69-80.

Hirschman E., Holbrook M.B. (1992), Postmodern Consumer Research - The Study of Consumption as Text. Newbury Park, California: Sage Publications.

Holbrook M.B. (1995), Consumer Research - Introspective Essays on the Study of Consumption, Sage Publications, Thousand Oaks.

Inhelder, B. et Piaget, J. (1966/2004). La psychologie de l'enfant. Paris: Quadrige.

John D. (1999), « Consumer Socialization of Children - A Retrospective look at twenty-five years of research », Journal of Consumer Research, 26 (3), 183-213.

Kline S. (2010), «Children as 'competent' consumers », Chapter 14 in Marshall D. (Ed.), Understanding Children as Consumers (pp. 239-257). Sage, London.

Lassare D., Roland-Lévy C. (1989), « Understanding children's economic socialization », in Grünert K.G. \& Olander F. (Eds.) Understanding economic behaviour (pp.347-368). Dordrecht (NL):Kluwer Academic Publishers.

Lave J., Wenger E. (1991), Situated learning: Legitimate peripheral participation, Cambridge UK: Cambridge University Press.

Moschis G., Churchill G. (1978), «Consumer socialization: a theoretical and empirical analysis », Journal of Marketing Research, 15, 599-609.

Moschis G., Moore R.L. (1979), «Decision-making among the young: a socialization perspective », Journal of Consumer Research, 6, 101-112. 
Nadel, J. et Baudonnière, P.-M. (1985). L'objet : moyen d'étude des compétences à communiquer. In P.M. Baudonnière(Ed.). Etudier l'enfant de la naissance à trois ans (pp. 139-163). Paris : CNRS, Coll. comportements.

Oestergaard P., Jantzen C. (2000), « Shifting perspectives in consumer research: from buyer behaviour to consumption studies », in Beckmann S.C. and Elliot R. (Eds.), Interpretive Consumer Research - Paradigms, Methodologies and Applications, Copenhagen Business School Press, Denmark.

Piaget, J. (1937/1967). La construction du réel chez l'enfant. Neuchâtel, Lausanne : Delachaux et Niestlé.

Rivière A. (1990), La psychologie de Vygotsky. Liège : Mardaga.

Rogoff B. (1998), «Cognition as a collaborative process », In D. Kuhn \& R.S Siegler (Eds.) Cognition and language (vol.2), Handbook of Child Psychology ( $5^{\text {th }}$ Edition) (pp. 679-738). New York: Wiley.

Rogoff B. (2003), The cultural nature of human development, Oxford: Oxford University Press.

Rogoff B., Moore L., Najafi B., Dexter A., Correa-Chavez M., Solis J. (2007), «Développement des repertoires culturels et participation des enfants aux pratiques quotidiennes », in Brougère G. \& Vandenbroeck M. (Dirs.). Repenser l'éducation des jeunes enfants, (pp. 103-138).Bruxelles : Peter Lang. Article original : (2006) Children's development of cultural repertoires through participation in everyday routines and practices, in Grusec J. and Hasting P. (Eds.). Handbook of Socialization, Guilford, New York.

Rogoff B., Wersch J.V. (Eds.), (1984), « Children's Learning in the 'Zone of Proximal Development' ", New Directions for Child Development. San Franscico: Jossey Bass Publishers.

Roland-Lévy C. (1990), «A cross-national comparison of Algerian and French children's economic socialization », Journal of Economic Psychology, 567-581.

Schneuwly B., Bronckart J.P. (Eds), Vygotsky aujourd'hui. Paris: Delachaux \& Niestlé.

Strauss A. (1952), « The development and transformation of monetary meanings in the child», American Sociological Review, 17, 275-286.

Vygotski L. (1934/1997), Pensée et langage, La dispute, Paris.

Ward S. (1974), «Consumer Socialization », Journal of Consumer Research, 1, 1-14.

Ward S., Wackam D., Wartella E., (1977), How Children Learn to Buy: the Development of Consumer Onformation-processing skills. Beverly Hills: Sage.

Ward S., Klees D.M., Wackman D.B., (1990), « Consumer socialization research: content analysis of post-1980 studies and some implications for future work », Advances in Consumer Research, 17, 798-803.

Wertsch J.V. (1985), Vygotsky and the social formation of mind, Cambridge MA: Harvard University Press. 